\title{
Factores familiares asociados con la prevalencia de Fluorosis dental en niños escolares en Cartagena-Colombia
}

\author{
Farith González Martínez ${ }^{a}$, Katherine Margarita Arrieta Vergara ${ }^{b}, N^{2}$ atalia Fortich Mesa ${ }^{c}$
}

\begin{abstract}
a Profesor y jefe del departamento de investigación programa de Odontología Universidad de Cartagena, Colombia.

b Profesora asociada departamento de Odontología Preventiva y Social, escuela de Odontología Universidad de Cartagena.

c Profesora y coordinadora de investigaciones escuela de Odontología Universidad Rafael Núñez, Cartagena.

Correspondencia: Farith González Martínez, barrio Zaragocilla, Campus de la salud, facultad de Odontología Universidad de Cartagena, Cartagena de Indias, Colombia. Tel. 095-6698173 ext. 115. Correo electrónico: farithgm@ hotmail.com- fgonzalezm1@ unicartagena.edu.co
\end{abstract}

Recibido el 23 de julio de 2012.

Aceptado para su publicación el 1 de septiembre de 2012.

\section{RESUMEN}

Objetivo. Evaluar la asociación entre prevalencia de Fluorosis dental y factores familiares responsables de la ingesta de fluoruros.

Diseño. Estudio de corte transversal.

Emplazamiento: Municipio de Cartagena de indias, Colombia.

Participantes. 588 binomios madre-hijo que consultaron en clínicas odontológicas universitarias entre los años 2009 y 2010.

Mediciones Principales. Se midió en niños la Fluorosis dental mediante el índice de Thylstrup y Fejerskov y los factores familiares responsables de la ingesta de fluoruros a través de una encuesta dirigida a las madres. Para el examen clínico se realizó estandarización intraexaminador e inter-examinador a partir de un observador clínico usado como referencia. La exploración de la asociación fue analizada con razones de disparidad e intervalos de confianza del $95 \%$. El análisis multivariable por regresión logística nominal, incluyendo factores con probabilidades menores de 0 .

Resultados. La prevalencia de fluorosis dental fue del 64,8 \%, siendo la clasificación leve la más frecuente. Mediante análisis multivariable, el mejor modelo mostró significancia estadística $(p<0,0001)$ para las variables frecuencia del cepillado dental tres veces al día $(O R=1,68)$, uso de dentífrico con alto contenido de flúor en ppm $(\mathrm{OR}=2,21)$ e ingesta de crema dental en momentos diferentes al cepillado $(\mathrm{OR}=1,65)$.

Conclusiones. Los factores familiares responsables del uso de crema dental con alto contenido de flúor fueron los de mayor peso para explicar la posible asociación con la alta prevalencia de Fluorosis en la población de estudio, lo que supone una interacción entre los efectos benéficos y secundarios del uso de fluoruros en la prevención de la caries.

Palabras clave. Fluorosis dental, Epidemiología, Factores familiares, Fluoruros.

\section{ABSTRACT}

Family Factors associated with Dental Fluorosis prevalence in school children Cartagena, Colombia

Objective. To assess the association between the prevalence of dental fluorosis and family factors responsible for fluoride intake

Design. Cross-sectional study

Location: Municipality of Cartagena de Indias, Colombia.

Participants. 588 mother-child pairings attending university dental clinics between the years 2009-2010

Main measurements. Measurement of Dental fluorosis in children using the Thylstrup and Fejerskov index and family factors responsible for fluoride intake by way of a survey aimed at mothers. For the clinical examination, intra- and inter-examiner standardization was conducted using a clinical observer as a reference. The exploration of the association was analysed with odds ratios and $95 \%$ confidence intervals. Multivariable analysis by nominal logistic regression, including factors with probabilities below 0 .

Results. The prevalence of dental fluorosis was $64,8 \%$, with the classification "slight" being the most frequent. By way of multivariable analysis, the best model showed statistical significance $(p<0,0001)$ for the variables: frequency of teeth brushing three times per day $(O R=1,68)$, use of toothpaste with high fluoride content in $\mathrm{ppm}(\mathrm{OR}=2,21)$ and the intake of toothpaste at times different from brushing $(\mathrm{OR}=1,65)$.

Conclusions. The family factors responsible for the use of toothpaste with a high fluoride content were those which gave the clearest explanation for a possible association with the high prevalence of fluorosis in the study population, which implies an interaction between the beneficial and secondary effects in the use of fluorides in the prevention of tooth decay.

Key words: Dental fluorosis, Epidemiology, Family Factors, Fluorides. 


\section{INTRODUCCIÓN}

Los Fluoruros han sido utilizados desde hace muchos años para la prevención de la caries dental. En EE.UU la Asociación Dental Americana (ADA), desde 1950, ha apoyado la fluoración del suministro de agua en las comunidades por considerarla un método seguro y eficaz para prevenir la caries dental. En 1953 se inició este procedimiento del agua potable en Colombia, pero algunas dificultades de costo-efectividad del programa motivaron el cambio de vehículo. En 1989 se comenzó con el programa de fluoración de la sal de consumo humano, el cual se ha mantenido con algunas dificultades para controlar las dosis óptimas en todas las marcas comerciales que existen en el mercado (dosis óptima=180-220ppm $\mathrm{F} / \mathrm{Kg} \mathrm{sal})^{1}$. Antes de esto ya se comercializaban en distintos países las pastas dentales con flúor y se inició la implementación de programas de enjuagues bucales con flúor en las escuelas.

Además de la utilización del flúor tópico acidulado en gel para aplicación profesional en las lesiones de caries, actualmente es reconocido que el uso del flúor, en cantidad y concentración adecuada, se constituye en un importante factor en la disminución de la ocurrencia de caries dental desde tempranas edades. Sin embargo, existe un problema de la sumatoria que se produce por las exposiciones a múltiples fuentes de fluoruros a las que nos vemos sometidos los seres humanos. Se trata de decisiones de salud pública usadas por los estados como medidas preventivas colectivas, entre las que se encuentran la ingesta diaria de agua potable y sal fluorada para el consumo². Además, existen otras fuentes que es posible controlar desde el núcleo familiar, si se tiene un conocimiento adecuado de sus consecuencias, entre las que se encuentran el consumo de bebidas como leche, té y vino y alimentos como pescados, algunos vegetales, cárnicos asados y frituras, así como los complementos de flúor usados por los Odontólogos en el consultorio y los que aporta la ingesta accidental de las cremas dentales durante el cepillado ${ }^{3,4}$. La sumatoria de estas exposiciones a fluoruros en todas estas fuentes, ocasiona probablemente una alta ingesta y posterior absorción por encima de los niveles óptimos (0.05$0.07 \mathrm{mg} \mathrm{F} / \mathrm{Kg} \mathrm{pc})^{5}$, la cual cuando se presenta por periodos prolongados a edad temprana causa toxicidad, manifestándose en la fijación del flúor especialmente en tejidos calcificados con la aparición de Fluorosis dental.
En muchos de los países, cuyas poblaciones disponen del servicio público de acueducto, se pueden garantizar niveles de flúor óptimos en el agua para el consumo humano por el continuo monitoreo que realizan las empresas públicas responsables de este control. Sin embargo, en aquellas regiones con un riesgo de ingesta de fluoruros superior al nivel óptimo es necesario evaluar las prevalencias de Fluorosis de forma permanente, debido a la ausencia de un control estatal sobre el consumo y al desconocimiento de la influencia de la ingesta de otras fuentes de fluoruros en el incremento del riesgo de Fluorosis dental5 ${ }^{5-7}$.

Actualmente muchos países de Latinoamérica y Europa han implantado políticas bucales de salud pública para realizar vigilancia epidemiológica de la Fluorosis dental. Sin embargo, en otros no existe claridad en las acciones para monitorear y controlar las diferentes exposiciones a los fluoruros $y$, todavía, no se tiene en cuenta el umbral de dosis acumulativa reconocido como la exposición total. Estos hechos apoyan la necesidad de nuevas investigaciones sobre la alteración de salud bucal, fundamentalmente en aquellos países en los cuales no existe claridad sobre la distribución demográfica de la Fluorosis dental y en los que presentan diferentes exposiciones a Fluoruros, que no han sido evaluadas dentro de los planes de salud pública ${ }^{5,6}$.

El objetivo del presente estudio fue evaluar la asociación entre prevalencia de Fluorosis dental y factores familiares responsables de la ingesta de fluoruros a partir de diferentes fuentes en niños escolares.

\section{MATERIAL Y MÉTODOS}

Se realizó un estudio descriptivo transversal en participantes binomios madre-hijo de la ciudad de Cartagena de Indias en Colombia. Fueron invitados a participar todos los niños entre 6 y 11 años que asistieron a consulta odontológica en las clínicas de tres instituciones universitarias, entre los años 2009 y 2010. El tamaño de la muestra fue de 588 sujetos, considerando la exploración de asociación con 12 factores familiares responsables de la ingesta de flúor, la frecuencia esperada de la Fluorosis reportada en la literatura ${ }^{7,8}$, error tipo I del $5 \%$, y una potencia del $80 \%$.

Para la selección de los participantes se utilizó un muestreo aleatorio simple en las clínicas Odontológicas de las instituciones (universidad 
de Cartagena, universidad del Sinú- Cartagena y universidad Rafael Núñez), durante el periodo de estudio. La selección fue realizada con ayuda de software estadístico STATA®, versión para Windows 12.0, obteniendo una serie de números aleatorios. Se procedió a evaluar el cumplimiento de los siguientes criterios: niños residentes en la ciudad de Cartagena durante los cinco primeros años de vida, presentar en completa erupción de uno o más pares homólogos de dientes indicados para el examen clínico y que sus padres aceptaran responder un cuestionario obteniendo su consentimiento informado por escrito. Por otro lado, se excluyeron a los niños con alguna malformación congénita que afectara huesos y dientes, los que recibieran en el momento del estudio tratamiento de ortodoncia $u$ ortopedia fija maxilar y aquellos que presentaran caries dental, obturaciones o fracturas dentales en la superficie vestibular examinada. Este proyecto fue avalado por el comité de ética institucional de la universidad de Cartagena, cumpliéndose las disposiciones internacionales (declaración de Helsinki 1964- modificación de Edimburgo 2000). Esta selección culminó en el segundo semestre del año 2010 y se realizó secuencialmente el examen clínico y las encuestas.

Las mediciones clínicas se realizaron mediante el índice Thylstrup y Fejerskov (TFI) ${ }^{9,10}$ y un instructivo para identificar los dientes patrones. Los exámenes fueron llevados a cabo en sillones dentales portátiles estándar y, para observar las lesiones, fue usado un espejo bucal plano y jeringas de aire en la superficie de los dientes, previa remoción de la placa dental mediante profilaxis profesional. Además, se utilizó una sonda periodontal para verificar si había discontinuidad en la superficie. Por otro lado, los factores familiares responsables de la ingesta de fluoruros se evaluaron a partir de un cuestionario estructurado, aplicado a las madres de los niños.

\section{Estandarización del examen clínico}

Se realizó una estandarización para evaluar la variabilidad inter-examinador e intra-examinador a partir de un observador clínico usado como punto de referencia. Para la selección del mejor examinador se aceptó un grado de acuerdo entre

\begin{tabular}{|c|c|}
\hline CÓDIGO & CARACTERÍSTICAS CLÍNICAS DEL ESMALTE \\
\hline 0 & $\begin{array}{l}\text { La traslucidez normal del esmalte brillante blanco cremoso permanece después de limpiar } \\
\text { y secar la superficie. }\end{array}$ \\
\hline 1 & $\begin{array}{l}\text { Se observan líneas delgadas opacas a lo largo de la superficie dental. En algunos casos } \\
\text { puede observarse en los bordes cuspídeos e incisales. }\end{array}$ \\
\hline 2 & $\begin{array}{l}\text { Las líneas opacas son más pronunciadas y forman áreas pequeñas dispersas a lo largo de } \\
\text { toda la superficie. Las opacidades en los bordes incisales y cuspídeos son más comunes. }\end{array}$ \\
\hline 3 & Ocurre fusión de las líneas opacas y hay áreas esparcidas opacas por toda la superficie. \\
\hline 4 & $\begin{array}{l}\text { La superficie completa exhibe una marcada opacidad con apariencia de tiza. Algunas } \\
\text { partes de las superficies expuestas a atrición o uso parecen menos afectadas. }\end{array}$ \\
\hline 5 & $\begin{array}{l}\text { La superficie completa es opaca con fosas redondeadas con perdida focal de esmalte } \\
\text { externo (menos de } 2 \mathrm{~mm} \text { de diámetro). }\end{array}$ \\
\hline 6 & $\begin{array}{l}\text { Las fosas emergen en el esmalte opaco y forman bandas de }<2 \mathrm{~mm} \text { de altura. Puede } \\
\text { observarse perdida de bordes cuspídeos de aprox. } 2 \mathrm{~mm}\end{array}$ \\
\hline 7 & $\begin{array}{l}\text { Hay pérdida de esmalte externo en áreas irregulares y menos de la mitad de la superficie } \\
\text { está involucrada. El esmalte remanente es opaco. }\end{array}$ \\
\hline 8 & $\begin{array}{l}\text { La pérdida de la porción superficial de esmalte involucra más de la mitad del mismo. El } \\
\text { esmalte restante es opaco. }\end{array}$ \\
\hline 9 & $\begin{array}{l}\text { Hay pérdida de la mayor parte de la superficie externa del esmalte dando como resultado } \\
\text { cambios en la forma anatómica del diente. }\end{array}$ \\
\hline
\end{tabular}

Tabla 1. Características clínicas del esmalte dental según TFI. 
0,75 y 0,85, a través del coeficiente de Kappa ponderado, obteniéndose como resultado a dos examinadores dentro de estos parámetros. El proceso de calibración fue realizado inicialmente con dientes permanentes exfoliados y fotografías $y$, después, con examen clínico in vivo para evaluar la concordancia entre la detección de los diferentes códigos del índice de TFI para Fluorosis dental.

\section{Detección de esmalte fluorótico}

$\mathrm{Se}$ inspeccionaron las superficies vestibulares, limpias y secas, y se determinó la presencia de la Fluorosis dental en seis pares de dientes (incisivos centrales y laterales y primeros molares en ambas arcadas). Luego se clasificó la apariencia macroscópica de los dientes en relación con la condición histológica del esmalte usando una escala ordinal con los indicadores ${ }^{9}(0$ a 9 ) (tabla 1 ).

\section{Cuestionario}

Se diseñó un cuestionario semi-estructurado y auto-suministrado a las madres de los participantes para evaluar su percepción en cuanto a las variables familiares relacionadas con la ingesta de fluoruros por parte de los niños. Este formato contenía 36 preguntas, 32 fueron estructuradas; 27 politómicas y cinco dicotómicas con única respuesta, además cuatro preguntas eran no estructuradas. Todas estas midieron las variables con los códigos y categorías observadas en la información

\begin{tabular}{|c|c|c|}
\hline VARIABLE & DIMENSIÓN & CÓDIGOS (CATEGORÍAS) \\
\hline $\begin{array}{l}\text { Características } \\
\text { demográficas }\end{array}$ & $\begin{array}{l}\quad \text { Edad (años) } \\
\text { Sexo } \\
\text { Estratificación social }\end{array}$ & $\begin{array}{c}1=6-7 ; 2=8-9 ; 3=10-11 \\
1=\text { masculino; } 2=\text { femenino } \\
1 \text { =bajo; } 2=\text { medio bajo; } 3=\text { medio; } 4=\text { medio } \\
\text { alto; } 5=\text { alto }\end{array}$ \\
\hline $\begin{array}{l}\text { Flúor en el agua para } \\
\text { consumo }\end{array}$ & $\begin{array}{c}\text { Origen del agua } \\
\text { Forma de consumo de agua }\end{array}$ & $\begin{array}{c}\text { 1=depósito subterráneo; } 2=\text { acueducto; } \\
3=\text { agua embotellada; } 4=\text { agua de estanques } \\
\text { móviles; } 5=\text { otros } \\
\text { 1=hervida; } 2=\text { depósito o estanques fijos; } \\
3=\text { grifo; } 4=\text { filtro; } 5=\text { otro }\end{array}$ \\
\hline $\begin{array}{l}\text { Flúor en la sal de } \\
\text { consumo }\end{array}$ & $\begin{array}{l}\text { Tipo de sal que consume } \\
\text { Cantidad de alimentos preparados con } \\
\text { sal durante el día } \\
\text { Cantidad de sal utilizada para la } \\
\text { preparación de alimentos en el día }\end{array}$ & 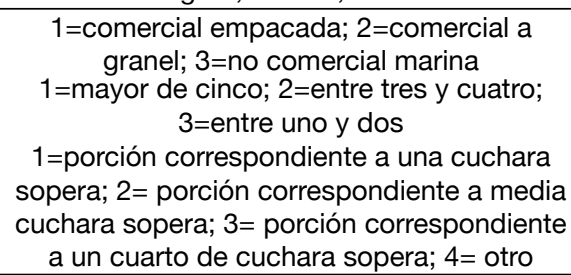 \\
\hline $\begin{array}{l}\text { Flúor como aplicación } \\
\text { profesional }\end{array}$ & $\begin{array}{l}\text { Edad de la primera aplicación de flúor } \\
\text { Cantidad de aplicaciones de flúor en } \\
\text { el año }\end{array}$ & $\begin{array}{c}1=\text { entre uno y tres años; } 2=\text { entre cuatro y } \\
\text { cinco años; } 3=\text { mayor de cinco años } \\
1=\text { cuatro o más; } 2=\text { =entre dos y tres; } 3=\text { una; } \\
4=\text { ninguna }\end{array}$ \\
\hline Flúor en pasta dental & $\begin{array}{c}\text { Utiliza pasta dental fluorada para el } \\
\text { cepillado } \\
\text { Edad de inicio del cepillado con pasta } \\
\text { dental fluorada } \\
\text { precuencia del cepillado dental con } \\
\text { pasta fluorada } \\
\text { Uso de dentífrico con alto contenido de } \\
\text { flúor, 1500ppm o más } \\
\text { Cantidad de dentífrico colocada en el } \\
\text { cepillo } \\
\text { Supervisión del cepillado del niño } \\
\text { Ingesta accidental de la crema dental } \\
\text { durante el cepillado } \\
\text { Ingesta de la crema dental en } \\
\text { momentos diferentes al cepillado }\end{array}$ & 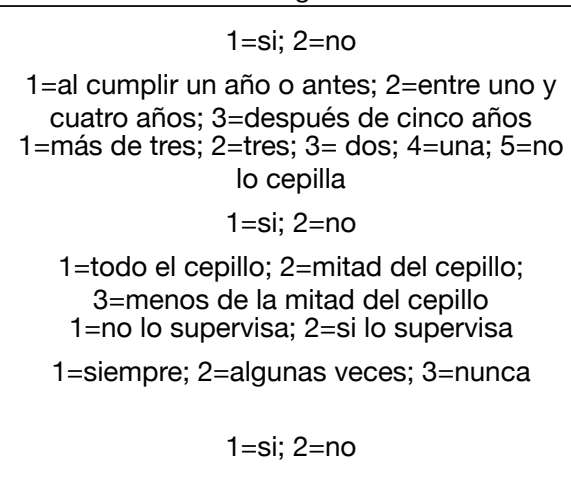 \\
\hline Flúor en alimentos & $\begin{array}{l}\text { Consume alimentos como pescados, } \\
\text { té, leche pulverizada, fresas, tomate } \\
\text { Cuál es la frecuencia de consumo de } \\
\text { estos alimentos } \\
\text { Desde que edad inició a consumir } \\
\text { alimentos fluorados }\end{array}$ & 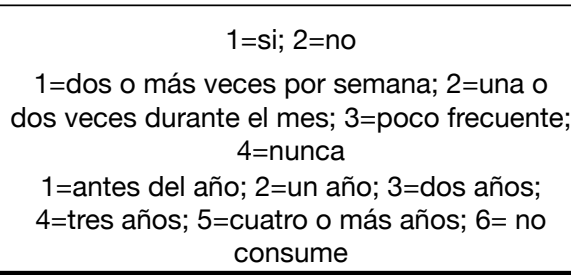 \\
\hline
\end{tabular}

Tabla 2. Cuestionario sobre ingesta de fluoruros aplicado a los participantes. 
complementaria (tabla 2). Este instrumento fue evaluado por tres jueces expertos con el fin de verificar la validez de apariencia y fue usado un análisis de confiabilidad-estabilidad, test-retest, aplicado en dos momentos diferentes de tiempo en un grupo piloto. Todos los encuestadores fueron entrenados para administrar los cuestionarios asignados. Por último, la información fue obtenida por examinadores cegados.

\section{Análisis estadístico}

Los datos fueron analizados a partir del programa STATA ${ }^{\circledR}$ (Stata Corp LP, College Station, TX, EE.UU.). Como variable dependiente usada en el análisis se tomó la presencia de Fluorosis dental a partir de los códigos 1-9 del TFI. Así mismo, la exploración de la asociación fue analizada a partir de las razones de disparidad (OR; IC 95\%). El análisis multivariable se realizó por regresión logística nominal, usando un procedimiento de pasos hacia atrás para incluir o excluir variables explicativas en el ajuste de los modelos, siendo seleccionadas las variables para los modelos finales sólo si tenían un valor de $p<0,05$ después del ajuste por la edad.

\section{RESULTADOS}

Un total de 657 madres y niños fueron invitados a participar en el presente estudio, 42 no cumplieron con los criterios de selección y 27 madres rehusaron a participar, obteniéndose 588 participantes con una tasa de respuesta del 95,9\%. Las madres que completaron el cuestionario tenían un promedio de edad de 31,5 años (DE: 6,8). Asimismo, el promedio de edad de los niños examinados fue de 8,3 años (DE: 1,25), siendo el grupo de edad más

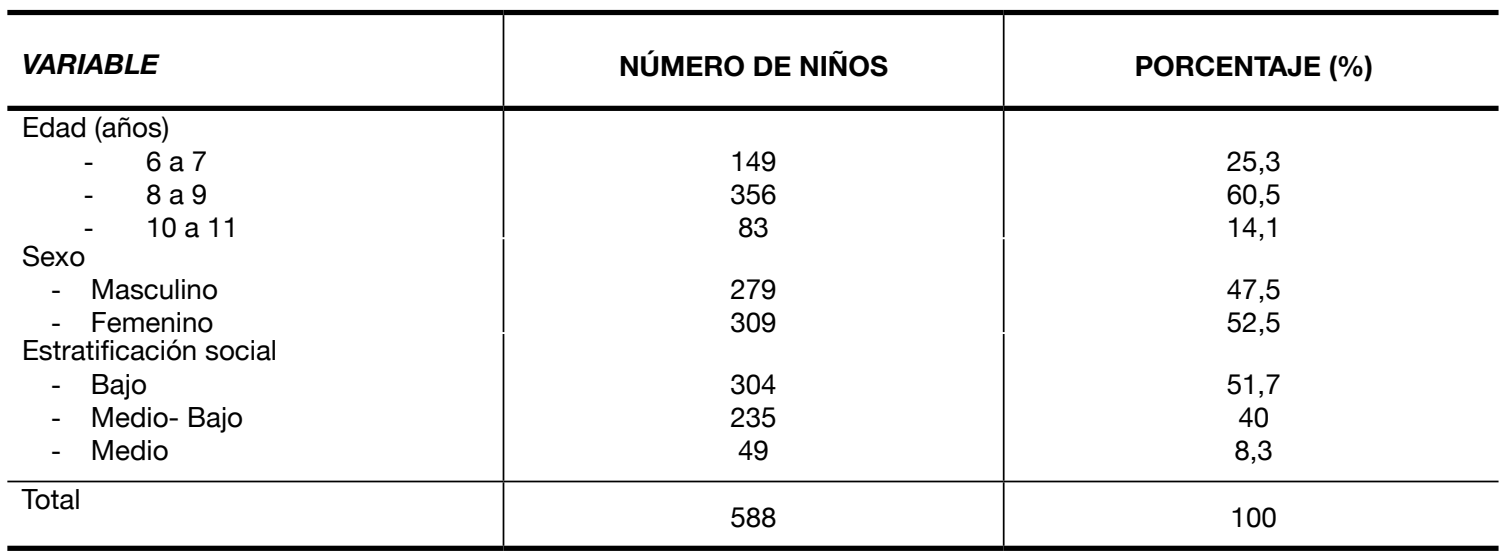

Tabla 3. Distribución de las características socio-demográficas de los participantes.

frecuente el de 8 a 9 años. En cuanto al sexo, los varones representaron el menor porcentaje. Todos los datos demográficos de los niños participantes se observan en la tabla 3.

La prevalencia de fluorosis dental en los participantes fue del 64,8\% (IC 95\%: 60,9-68,7), incluyendo los códigos 1-9 TFI, siendo muy similar para la edad y el sexo. De acuerdo a la gravedad de la Fluorosis dental, fueron más frecuentes las superficies dentales identificadas con códigos TFI 1-2, mientras que las superficies de mayor severidad identificadas con códigos TFI iguales o superiores a 5 se observaron en menos de $1 \%$ (tabla 4 y 5). Respecto a la presencia de los factores familiares responsables de la ingesta de fluoruros, los de mayor prevalencia fueron el uso de dentífricos con alto contenido de flúor en ppm, consumo de alimentos con alto contenido de flúor, cantidad de dentífrico usada entre la mitad y todo el cepillo y preparación con sal entre tres y cinco tipos de alimentos al día. Los otros factores aparecen en la tabla 6.

En la tabla 7 se presentan los resultados del análisis multivariable por regresión logística con estimadores ajustados por edad. El mejor modelo mostró significancia estadística para tres factores, todos provenientes de la fuente de flúor en las pastas dentales, que fueron frecuencia del cepillado dental tres veces al día, uso de dentífrico con alto contenido de flúor en ppm e ingesta de pasta dental en momentos diferentes al cepillado $(P=0.000)$. 


\begin{tabular}{|c|c|c|c|c|}
\hline $\begin{array}{l}\text { FLUOROSIS } \\
\text { DENTAL }\end{array}$ & $\begin{array}{c}\text { 6-7 AÑOS } \\
(n=149)(\% ; \text { IC 95\%) }\end{array}$ & $\begin{array}{c}\text { 8-9 AÑOS (\%) } \\
(n=356)(\% ; \text { IC 95\%) }\end{array}$ & $\begin{array}{c}\text { 10-11 AÑOS } \\
(n=83)(\% ; \text { IC 95\%) }\end{array}$ & $\begin{array}{c}\text { TODOS LOS GRUPOS } \\
(n=588)(\% ; \text { IC } 95 \%)\end{array}$ \\
\hline $\begin{array}{l}\text { f0 } \\
f 1 \\
\text { f2 } \\
\text { f3 } \\
\text { F4 }\end{array}$ & $\begin{array}{c}58(38,9 ; 31,1-46,8) \\
70(46,9 ; 38,9-55,0) \\
21(14,1 ; 8,5-19,7) \\
0(0,0 ;--) \\
91(61,1 ; 53,2-68,9)\end{array}$ & $\begin{array}{c}122(34,3 ; 29,3-39,2) \\
164(46,1 ; 40,9-51,3) \\
67(18,8 ; 14,7-22,9) \\
3(0,84 ; 0,01-1,7) \\
234(65,7,60,8-70,7)\end{array}$ & $\begin{array}{c}27(32,5 ; 22,4-42,7) \\
40(48,2 ; 37,4-59,0) \\
15(18,1 ; 9,7-26,4) \\
1(1,20 ; 0,1-3,69) \\
56(67,5 ; 57,3-77,6)\end{array}$ & $\begin{array}{c}207(35,2 ; 31,3-39,1) \\
274(46,6 ; 42,6-50,6) \\
103(17,5 ; 14,4-20,6) \\
4(0,68 ; 0,01-1,3) \\
381(64,8 ; 60,9-68,7)\end{array}$ \\
\hline
\end{tabular}

Tabla 4. Porcentaje de niños de acuerdo al tipo y la gravedad de Fluorosis (TFI), en todos los grupos de edad. f0 número total de niños sin lesiones fluoróticas (TFI código 0); f1 número total de niños con lesiones fluoróticas en esmalte (TFI códigos 1-2); f2 número total de niños con lesiones fluoróticas en esmalte (TFI códigos 3-4); f3 número total de niños con lesiones fluoróticas (TFI códigos 5 o más); f4 número total de niños con lesiones fluoróticas en todos los códigos (TFI códigos 1-9).

\begin{tabular}{l|cc}
\hline Fluorosis Dental & $\begin{array}{c}\text { Masculino } \\
(\mathrm{n}=279)(\% ; \mathrm{IC} 95 \%)\end{array}$ & $\begin{array}{c}\text { Femenino } \\
(\mathrm{n}=309)(\% ; \mathrm{IC} 95 \%)\end{array}$ \\
\hline $\mathrm{f0}$ & $89(31,9) 26,4-37,4$ & $118(38,2) 32,8-43,6$ \\
$\mathrm{f} 1$ & $132(47,3) 41,4-53,2$ & $142(46,0) 40,4-51,5$ \\
$\mathrm{f} 2$ & $55(19,7) 15,0-24,4$ & $48(15,5) 11,5-19,6$ \\
$\mathrm{f} 3$ & $3(11) 0,1-2,3$ & $1(0,32) 0,03-0.9$ \\
$\mathrm{f} 4$ & $190(68,1) 62,6-73,6$ & $191(61,8) 56,4-67,2$ \\
\hline
\end{tabular}

Tabla 5. Porcentaje de niños de acuerdo al tipo y la gravedad de Fluorosis (TFI), en todos los grupos por sexo. f0 número total de niños sin lesiones fluoróticas (TFI código 0); f1 número total de niños con lesiones fluoróticas en esmalte (TFI códigos 1-2); f2 número total de niños con lesiones fluoróticas en esmalte (TFI códigos 3-5); f3 número total de niños con lesiones fluoróticas (TFI códigos 5 o más); f4 número total de niños con lesiones fluoróticas en todos los códigos (TFI códigos 1-9).

\begin{tabular}{|c|c|c|c|}
\hline $\begin{array}{l}\text { FACTORES FAMILIARES RELACIONADOS CON LA INGESTA } \\
\text { DE FLÚOR }\end{array}$ & $\begin{array}{l}\text { Número } \\
\mathrm{N}=580\end{array}$ & $\begin{array}{l}\text { Prevalencia } \\
\text { (\%) }\end{array}$ & IC 95\% \\
\hline $\begin{array}{l}\text { Consumo de agua en depósitos subterráneos } \\
\text { Consumo de agua hervida } \\
\text { Prepara con sal entre tres y cinco tipos de alimentos al día } \\
\text { El niño ha recibido tres o más aplicaciones de flúor en el año } \\
\text { Utiliza crema dental fluorada para el cepillado del niño } \\
\text { El inicio del cepillado dental del niño fue al año o antes } \\
\text { La frecuencia del cepillado dental del niños es tres veces al día } \\
\text { Usa dentífrico con alto contenido de flúor para el cepillado del } \\
\text { niño } \\
\text { No supervisa el cepillado dental del niño } \\
\text { Utiliza cantidad de dentífrico entre la mitad y todo el cepillo } \\
\text { El niño ingiere crema dental durante el cepillado } \\
\text { El niño ingiere crema dental en momentos diferentes al cepillado } \\
\text { Consumo de alimentos fluorados dos o más veces por semana }\end{array}$ & $\begin{array}{c}9 \\
233 \\
344 \\
57 \\
308 \\
324 \\
292 \\
448 \\
\\
234 \\
398 \\
302 \\
255 \\
444\end{array}$ & $\begin{array}{l}1,5 \\
39,6 \\
58,5 \\
9,6 \\
52,4 \\
55,1 \\
49,7 \\
76,2 \\
39,8 \\
67,7 \\
51,4 \\
43,4 \\
75,5\end{array}$ & $\begin{array}{r}0,05-2,5 \\
35,7-43,6 \\
54,5-62,5 \\
7,3-12,1 \\
48,3-56,4 \\
51,1-59,1 \\
45,6-53,7 \\
72,7-79,6 \\
35,8-43,8 \\
63,9-71,5 \\
47,3-55,4 \\
39,3-47,4 \\
72,0-79,0\end{array}$ \\
\hline
\end{tabular}

Tabla 6. Ocurrencia de Factores familiares responsables de la ingesta de fluoruros en niños escolares de ciudad de Cartagena 2010. 


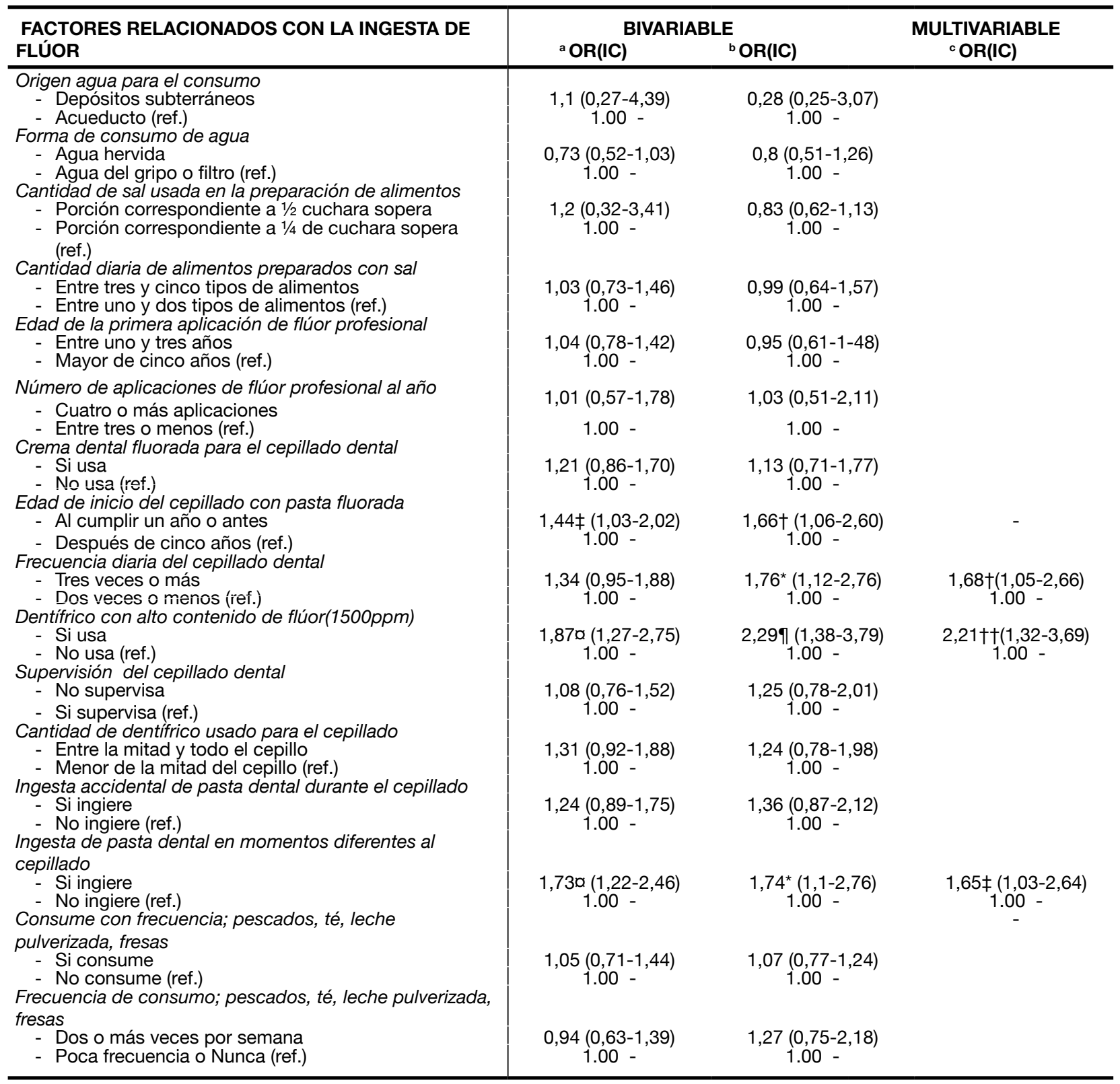

Tabla 7. Análisis de regresión logística para fluorosis y factores familiares responsables de la ingesta de fluoruros en niños escolares, Cartagena 2010. Ref.=categoría de referencia; ${ }^{a}$ OR razón para Fluorosis sin ajustar (TFI 1-9); b OR razón para Fluorosis ajustada por edad (TFI 1-9); ${ }^{\circ}$ OR modelo de regresión para Fluorosis (TFI 1-9) $\left(X^{2}=20,1 ; P=0.000\right)$; valores de probabilidad con significancia estadística; $\dagger P=0.02$, $¥ \mathrm{P}=0.03,{ }^{*} \mathrm{P}=0.01$, ๆ $\mathrm{P}=0.001,0 \mathrm{P}=0.002, \dagger \dagger \mathrm{P}=0.003$

\section{DISCUSIÓN}

Los resultados del presente estudio, aunque provienen de mediciones transversales en donde no es posible garantizar la temporalidad, porque se utilizan cuestionarios que exploran la asociación con determinantes y los resultados pueden tener limitantes como la subestimación de algunas exposiciones que cambian con el tiempo y el efecto de memoria de los sujetos evaluados, sí pueden aproximarnos al entendimiento de la distribución de la Fluorosis en la población de estudio, así como de posibles factores familiares responsables de la ingesta de fluoruros desde diferentes fuentes. Todo ello, podría contribuir a la implementación de herramientas preventivas que logren evitar las exposiciones a los fluoruros de más alto peso, para en un futuro disminuir la morbilidad bucal, que representa esta alteración para las regiones que tienen diferentes fuentes de exposición como al agua y sal de consumo ${ }^{11}$, así como alimentos, bebidas y cremas dentales fluoradas. 
Al usar el índice de Fluorosis dental TFI, se logra una importante aproximación a la detección del incremento en la porosidad de la superficie y en la sub-superficie del esmalte, a través de identificación de manchas blancas y opacas y en casos más avanzados estriaciones moteadas de color amarillo o marrón y hendiduras transversales en la superficie esmaltada del diente ${ }^{10}$. Éste índice comprende una escala ordinal y describe de manera detallada los cambios macroscópicos en el diente, en relación con los cambios histológicos que ocurren en el esmalte como producto de una ingesta excesiva de fluoruros. Esto permitió evidenciar aspectos de la enfermedad desde todos sus estadios incluyendo las primeras etapas, lo cual es posible reconocer con alta validez.

Según los resultados encontrados en el presente estudio, la prevalencia de fluorosis dental fue alta, siendo la clasificación leve la de mayor frecuencia. Sin embargo, esta ocurrencia hay que tenerla en cuenta, especialmente cuando los sujetos evaluados han tenido acceso a los servicios de salud bucal en clínicas odontológicas y se supone que sus padres tienen un adecuado conocimiento sobre las diferentes exposiciones relacionadas con la Fluorosis dental y deben evitarlas. No obstante esta alteración ha tenido un aumento significativo en los últimos años en la población general, lo cual se ha producido probablemente por la exposición indiscriminada a diferentes fuentes de fluoruros, las cuales son recomendadas para la prevención de la caries dental, siendo considerado este riesgo de mayor prioridad para la salud pública a nivel bucal. También se ha observado prevalencias altas también en poblaciones con similares características ${ }^{12-14}$, lo que demuestra que la tendencia es más global, especialmente en regiones donde existe también alta incidencia de caries dental.

Respecto a los resultados mostrados en el análisis multivariable, las exposiciones originadas por el uso de crema dental con alto contenido de flúor (frecuencia del cepillado tres veces al día, uso de dentífrico con alto contenido de flúor en ppm y la ingesta de crema dental en momentos diferentes al cepillado) fueron las de mayor fuerza estadística para explicar la presencia de fluorosis. No obstante, la fuente de agua potable fluorada para el consumo diario y el uso de suplementos de flúor aunque han sido reportados en la literatura como los principales riesgos para fluorosis dental ${ }^{15}$, no fueron estadísticamente significativas en el presente estudio. Esto podría deberse a que las familias de clase social baja con hijos menores de tres años disponen como única medida preventiva de caries dental del acceso a cremas dentales con alto contenido en flúor, cuyos efectos secundarios son conocidos $^{16}$, especialmente si estas cremas son ingeridas. Por otra parte, se han confirmado los beneficios sobre la prevención de caries dental con la utilización de cremas dentales con contenido moderado de flúor en $\mathrm{ppm}^{17,18}$.

Estos hallazgos confirman que los niños en edades tempranas se exponen a diferentes fuentes de fluoruros, las cuales son recomendadas por Odontólogos y utilizadas por sus padres para evitar la presencia de la caries dental. Asimismo, se ha demostrado que los niños entre 1,5 y 2,5 años de edad ingieren entre el $64,3 \%$ y el $83,9 \%$ de la crema dental que utilizan ${ }^{19}$, e incluso a los cinco años de edad se ingiere alrededor del $30 \%$ durante esta actividad $^{20,21}$. Por otro lado, el riesgo se incrementa si además de la ingesta del dentífrico, la frecuencia del cepillado es mayor a dos veces al día, si realizan el cepillado con crema dental con alto contenido de flúor (>1,200 ppm) o si se coloca gran cantidad de dentífrico en el cepillo'. La exposición a fluoruros a través de las cremas dentales, ha sido reportada en numerosos estudios como riesgo para fluorosis. Tabari et al. ${ }^{22}$ y Mascarenhas et al ${ }^{23}$. reportaron que los niños que usaban cremas dentales fluoradas (>1,200 ppm de flúor) mostraron entre 1,6 y 1,83 veces mayor riesgo respecto a los que usaron cremas dentales con baja concentración de flúor.

Los resultados del presente estudio permiten aproximarnos al conocimiento del riesgo familiar responsable de la ingesta de fluoruros asociado a fluorosis dental y pueden ser útiles para la prevención de esta alteración en poblaciones con características similares. En este sentido, ciertos hábitos familiares de ingesta de fluoruros de diferentes fuentes deberían controlarse a temprana edad, incidiendo directamente desde el núcleo familiar en la disminución de los efectos secundarios de las medidas preventivas usadas para la caries dental, considerados como manifestaciones clínicas de relevancia estética y funcional para la calidad de vida de los individuos que los presentan.

Se concluye que los factores familiares responsables de la ingesta de crema dentales (frecuencia del cepillado tres veces al día, uso de dentífrico con alto contenido de flúor en ppm y la ingesta de crema dental en momentos diferentes al cepillado) fueron los de mayor fuerza estadística para explicar la posible asociación con la alta prevalencia de Fluorosis en la población de estudio, 
lo que podría considerarse como una interacción entre los efectos benéficos y secundarios del uso de herramientas para la prevención de la caries dental.

\section{AGRADECIMIENTOS}

A todo el personal académico y administrativo de las instituciones universitarias que participaron en el presente estudio (universidad de Cartagena, universidad del Sinú- Cartagena y universidad Rafael Núñez) y a los investigadores integrantes del grupo de Salud Pública de la Universidad de Cartagena GISPOUC.

\section{BIBLIOGRAFÍA}

1. Franco $A M$, Martignon $S$, Saldarriaga $A$, González $M C$, Arbeláez MI, Ocampo A, Luna LM, Martínez- Mier EA, Villa AE. Total fluoride intake in children aged 22-35 months in four Colombian cities. Community Dent Oral Epidemiol. 2005;33:1-8.

2. Bulnes-López RM, Ramón-Frías $T$, Bermúdez-Ocaña $D$, Isela Juárez-Rojop, Borbolla-Sala ME, Piña-Gutiérrez OE. Identificación de fluorosis dental en una población estudiantil universitaria en el Estado de Tabasco, México. Salud En Tabasco. 2008;14 (3):776-788.

3. José-María García-Camba de la Muela, Felisa GarcíaHoyos, Margarita Varela Morales, Ángel González Sanz. Absorción sistémica de flúor en niños secundaria al cepillado con dentífrico fluorado. Rev Esp Salud Pública. 2009; 83 (3):415-425.

4. Franklin Delano Soares Forte, Suzely Adas Saliba Moimaz, Fábio Correia Sampaio. Urinary Fluoride Excretion in Children Exposed to Fluoride Toothpaste and to Different Water Fluoride Levels in a Tropical Area of Brazil. Braz Dent J. 2008; 19(3):214-218.

5. Petersen PE. The world oral health report 2003: continuous improvent of oral health in the 21st century; the approach of the WHO Global Oral Health Programme. Community Dent Oral Epidemiol. 2003; 31(Supl 1):3-23.

6. DesBesten PK, Keels MA, Heller KE. The effect of dental fluorosis to the use of fluoride supplements. Community Dent Oral Epidemiol. 1999; 27:41-7.

7. Cunha-Cruz J, Nadanovsky P. Dental Fluorosis. Increases caries risk. Journal of evidence based dental practice. 2005; 5:170-171.

8. Villena RS. An investigation of the transverse technique of dentifrice application to reduce the amount of fluoride dentifrice for young children. Pediatr Dent. 2000; 22:312-7.

9. Fejerskov Larsen MJ, Richards A, Baelum V. Dental tissue effects of fluoride. Adv Dent Res. 1994; 8(1):15-31.

10. Thylstrup A, Fejerskov O. Clinical appearance of dental fluorosis in permanent teeth in relation to histologic changes. Community Dent Oral Epidemiol. 1978; 6:315-328.

11. Vallejos-Sánchez A, Medina-Solís CE, Casanova-Rosado JF, Maupomé G, Minaya-Sánchez M, Pérez Olivares S. Dental fluorosis in cohorts born before, during and after the national salt fluoridation program in a community in Mexico. Acta Odontol Scand. 2006; 64:209-213.
12. Cochran JA, Ketley CE, Árnadóttir IB, Fernandes B, KoletsiKounari $\mathrm{H}$, Oila A-M, et al. A comparison of the prevalence of fluorosis in 8-year-old children of seven european study sites a standardized methodology. Community Dent Oral Epidemiol. 2004; 32 (suppl 1):28-33.

13. Ramírez B, Franco Á, Ochoa E. Fluorosis Dental en Escolares de 6 a 13 Años de Instituciones Educativas Públicas de Medellín, Colombia. 2006. Rev. Salud Pública (Bogotá). 2009; 11 (4):631-640.

14. Bottenberg P, Deeclerck D, Ghidey W, Bogaerts K, Vanobbergen J, Martens L. Prevalence and determinants of enamel fluorosis in Flemish schoolchildren. Caries Res. 2004; 38:20-28.

15. Abanto J, Rezende K, Salazar S, Alves F, Celiberti P, Ciamponi A. Dental fluorosis: Exposure, prevention and managment. Med oral Patol Oral Cir Bucal. 2009; 14(2):103-7.

16. Wong MC, Clarkson J, Glenny AM, Lo EC, Marinho VC, Tsang BW, Walsh T, Worthington HV. Cochrane reviews on the benefits/risks of fluoride toothpastes. J Dent Res. 2011; 90:573-

17. TJ. Lima, CCC. Ribeiro, LMA. Tenuta, JA. Cury. Low-Fluoride Dentifrice and Caries Lesion Control in Children with Different Caries Experience: A Randomized Clinical Trial. Caries Res. 2008;42:46-50.

18. JA. Tavener, GM. Davies, RM. Davies, RP. Ellwood. The Prevalence and Severity of Fluorosis in Children Who Received Toothpaste Containing either 440 or 1,450 ppm F from the Age of 12 Months in Deprived and Less Deprived Communities. Caries Res. 2006;40:66-72.

19. O'Mullane DM, Cochran JA, Whelton HP. Fluoride ingestion from toothpaste: background to European Union-funded multi-centre project. Community Dent Oral Epidemiol. 2004; 32(Suppl 1):5-8.

20. Puppin-Rontani RM, Kassawara AB, Rodriguez CR. Influence of socioeconomic level and dentrifice brand on the oral higiene habits and fluoride dentifrice ingestion. J Clin Pediar Dent. 2002; 26:319-325.

21. Cochran JA, Ketley CE, Duckworth RM, Van Loveren C, Holbrook WP, Seppä L, et al. Development of a standardize method for comparing fluoride ingested from toothpaste by 1.5-3.5 years-old children in seven European countries. Part 2: Ingestion result. Community Dent Oral Epidemiol. 2004; 32 (supl 1):47-53.

22. Tabari ED, Ellwood R, Rugg-Gunn AJ, Evans DJ, Davies RM. Dental fluorosis in permanent incisor teeth in relation to water fluoridation, social deprivation and toothpaste use in infancy. Br Dent J. 2000; 189:216-20.

23. Mascarenhas AK. Risk factor for dental fluorosis: a review of the recent literature. Pediatr Dent. 2000; 22:269-77. 